\title{
Infecção VIH e Neoplasias Não Definidoras de SIDA: Experiência de um Centro
}

\author{
HIV Infection and Non-AIDS-Defining Malignancies: An Outpatient Clinic \\ Experience
}

\author{
Maria do Carmo FEVEREIRO ${ }^{1}$ \\ Acta Med Port 2014 Mar-Apr;27(2):181-190
}

RESUMO

Introdução: Os doentes infectados pelo Vírus da Imunodeficiência Humana têm um risco elevado de desenvolver diferentes tipos de Neoplasias. Com a introdução da terapêutica anti-retroviral de alta potência, e consequente aumento da sobrevida, assistimos a uma mudança do espectro das patologias relacionadas com a infecção, nomeadamente das doenças Oncológicas, com aumento das Neoplasias Não Definidoras em deterimento das Definidoras de SIDA.

Material e Métodos: Caracterização dos doentes com infecção Vírus da Imunodeficiência Humana e diagnóstico de Neoplasias Não Definidoras acompanhados ao longo de 16 anos na Consulta de Medicina/Imunodeficiência do Hospital de São José, através da consulta dos processos clínicos e avaliação retrospectiva dos aspectos demográficos, epidemiológicos, clínico-laboratoriais, tratamento e sobrevida.

Resultados: Nos 1042 doentes avaliados, foram identificados 34 casos de Neoplasias Não Definidoras, principalmente em homens (78\%) e com idade mediana de 55 anos. As neoplasias mais frequentes foram: pulmão $(20,6 \%)$, bexiga $(17,6 \%)$, próstata $(8,8 \%)$ e canal anal $(5,9 \%)$, sendo o tempo médio entre o diagnóstico da infecção pelo Vírus da Imunodeficiência Humana e da Neoplasias Não Definidoras de 6,8 \pm 4 anos. Na altura do diagnóstico da Neoplasias Não Definidoras a maioria dos doentes $(78,8 \%)$ estava sob terapêutica anti-retroviral de alta potência, em média desde há 5,7 \pm 3 anos, encontrando-se imunovirologicamente controlada. No total verificaram-se $45,5 \%$ óbitos, sobretudo em doentes com Neoplasia do pulmão $(20 \%)$.

Conclusão: Perante o risco de desenvolvimento de Neoplasias Não Definidoras nos doentes infectados pelo Vírus da Imunodeficiência Humana, torna-se fundamental o investimento em estratégias de prevenção, promoção de cessação tabágica e vacinação, bem como aplicação de protocolos de rastreio ajustados a esta população.

Palavras-chave: Infecção por VIH; Neoplasias; Síndrome de Imunodeficiência Adquirida; Terapêutica Anti-Retroviral.

\section{ABSTRACT}

Introduction: Human Immunodeficiency Virus infected patients have an increased risk for developing different types of cancer. After the introduction of highly active antiretroviral therapy (HAART), and consequent increased survival, a shift has been seen in the spectrum and evolution of HIV infection related diseases, particularly oncological ones, with a tendency to increase non-AIDS-defining malignancies (NADM) as opposed to AIDS defining malignancies.

Material and Methods: Characterization of the Human Immunodeficiency Virus infected patients with a non-AIDS defining malignancy diagnosis, followed over 16 years at an outpatient clinic in Lisbon through the review of medical records and retrospective evaluation of demographic, epidemiological, clinical and laboratorial parameters, treatment and mortality.

Results: Of the 1042 patients evaluated, there were 34 Non-AIDS defining malignancy cases identified, mostly in men (78\%), with a median age of 55 years. The most common cancers were: lung (20.6\%), bladder (17.6\%), prostate (8.8\%), and anal carcinoma (5.9\%). The mean time between Human Immunodeficiency Virus infection and non-AIDS-defining malignancy diagnosis was $6.8 \pm 4$ years. At the time of non-AIDS- defining malignancy diagnosis the majority of patients $(78.8 \%)$ was receiving HAART for a mean period of $5.7 \pm$ 3 years, most of whom were immune and virologically controlled (64\%). There were $45.5 \%$ deaths, mainly in patients with lung cancer $(20 \%)$.

Conclusion: Given the risk of developing a non-AIDS-defining malignancy in Human Immunodeficiency Virus-infected patients, it is essential to continue to invest in prevention strategies, promote smoking cessation as well as vaccination programs, as well as applying screening protocols adjusted to this population.

Keywords: Acquired Immunodeficiency Syndrome; Antiretroviral Therapy, Highly Active; HIV Infections; Neoplasms.

\section{INTRODUÇÃO}

Os doentes infectados por Vírus de Imunodeficiência Humana (VIH) têm um risco elevado, superior ao da população em geral, para o desenvolvimento de diferentes tipos de neoplasias. ${ }^{1-4}$ Com a introdução da terapêutica anti-retroviral de alta potência (TARV), e consequente aumento da sobrevida, assistimos a uma mudança do espectro e evolução das patologias relacionadas com a infecção por VIH, nomeadamente das doenças oncológicas, com tendência para o aumento das Neoplasias Não Definidoras (NNDS) em deterimento das Definidoras de SIDA (NDS). ${ }^{2-8}$ Revi- sões anteriores apontam para uma frequência das neoplasias entre duas e quatro vezes superior à população geral, com uma incidência variável de acordo com idade, sexo, raça, tipo de neoplasia e região geográfica, sendo as mais frequentes: o linfoma de Hodgkin, neoplasia do pulmão, e os carcinomas relacionados com vírus oncogénicos como o carcinoma hepatocelular e neoplasia do canal anal. ${ }^{3,4,9,10}$ As NNDS geralmente ocorrem em doentes mais jovens, têm uma apresentação atípica e mais agressiva, condicionando uma rápida progressão da doença, com altas taxa de reci-

1. Serviço de Medicina 1.4. Hospital São José. Centro Hospitalar Lisboa Central. Lisboa. Portugal.

Recebido: 07 de Junho de 2013 - Aceite: 11 de Fevereiro de 2014 | Copyright @ Ordem dos Médicos 2014 
diva e má resposta à terapêutica. ${ }^{11-13}$

No que diz respeito à etiopatogenia, os dados são ainda escassos e controversos. Tal como na população geral, parecem estar envolvidos alguns comportamentos e estilos de vida (tabagismo, alcoolismo, exposição solar). ${ }^{7,14}$ Menos bem compreendida é a influência do estado de imunossupressão, semelhante ao que se verifica em doentes transplantados ou sob terapêuticas imunossupressoras cróni-

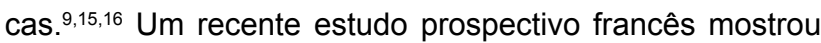
que o risco de neoplasia aumenta progressivamente para valores de Linfócitos T CD4+ inferiores a 500 células $/ \mathrm{mm}^{3}$ sendo o risco ainda maior se inferiores a 50 células $/ \mathrm{mm}^{3} .^{15}$ Por outro lado, um estudo australiano que envolveu doentes transplantados demonstrou um risco acrescido e com padrão semelhante de NNDS nestes doentes, sugerindo ser a própria imunossupressão a responsável pelo aumento desse risco. ${ }^{16}$ Adicionalmente, o próprio $\mathrm{VIH}$ parece exercer uma acção directa em diversos mecanismos celulares contribuindo para o desenvolvimento de neoplasias. Alguns desses mecanismos incluem a activação de proto-oncogenes, interferência na regulação do ciclo celular, inibição de genes supressores de tumores, entre outros. ${ }^{12,17}$ Também a co-infecção com outros vírus oncogénicos (Hepatites $B$ e C, Vírus Epstein-Barr - EBV, e Vírus do Papiloma Humano - HPV) potencia o desenvolvimento de neoplasias, pelo aumento da sua replicação viral e persistência de infecção, que condicionam uma mais rápida progressão da sua história natural. ${ }^{16,18,19}$ Por último, a utilização de terapêutica anti-retroviral, particularmente dos inibidores não nucleósidos da transcriptase reversa (INNTR), tem sido apontada como potencial factor carcinogénico adicional. ${ }^{9,20}$

Apesar de as neoplasias constituírem, actualmente, uma importante causa de mortalidade nos doentes infectados por $\mathrm{VIH},{ }^{21}$ na maioria dos casos não há recomendações específicas para o rastreio e tratamento precoces. ${ }^{19}$ No caso da European AIDS Clinical Society, estão recomendados alguns rastreios nomeadamente para as neoplasias do canal anal, mama, colo do útero, colo-rectal, fígado e próstata. ${ }^{22}$ Neste contexto, a prevenção passa sobretudo pelas campanhas de informação, promoção de cessação tabágica e vacinação. ${ }^{2}$

Relativamente ao tratamento das patologias oncológicas nestes doentes, geralmente não difere do tratamento da população geral, no entanto há que ter em consideração não só a localização primária, como o estado geral do doente, possíveis interacções medicamentosas e toxicidades cumulativas. ${ }^{12}$

No sentido de contribuir para o conhecimento e caracterização das NNDS, bem como eventuais factores de risco, segue-se a caracterização de um grupo de doentes com infecção por VIH e posterior diagnóstico de Neoplasia Não Definidora de SIDA, seguidos ao longo de 16 anos na Consulta de Medicina/Imunodeficiência do Hospital de São José - Lisboa, Portugal.

\section{MATERIAL E MÉTODOS}

Caracterização da população de doentes com infec- ção por VIH e diagnóstico de Neoplasia Não Definidora de SIDA acompanhados ao longo de 16 anos (1997 - 2012) na Consulta de Medicina/Imunodeficiência do Hospital de São José, CHLC-EPE - através da consulta de uma base de dados informática e dos processos clínicos em papel, e avaliação retrospectiva dos seguintes parâmetros: sexo, idade, raça, tipo de VIH e categoria de transmissão, condição imunológica e virológica, eventuais factores de risco para o desenvolvimento de neoplasia (hábitos tabágicos, hábitos etanólicos, co-infecção com HPV e vírus de hepatites B e C), tempo de evolução da infecção por VIH até ao diagnóstico de neoplasia, presença, tipo e duração de terapêutica anti-retroviral (TARV) até ao diagnóstico de neoplasia, tipo de neoplasia e sua histologia, tipo de tratamento (cirurgia, quimioterapia, radioterapia, hormonoterapia ou outros) e óbitos.

Para este estudo foram excluídos os doentes com antecedentes de patologia oncológica prévia ao diagnóstico da infecção por VIH, bem como os que abandonaram o seguimento ou foram transferidos para outra consulta sem diagnóstico confirmado de neoplasia. O diagnóstico de neoplasia foi feito com base na suspeição clínica bem como suporte clinico-laboratorial, radiológico e/ou histopatológico.

De salientar a dificuldade na obtenção dos dados, com algumas lacunas no registo da informação clínica parcialmente não informatizada.

Para comparar a sobrevivência entre grupos, foi utilizado o estimador de Kaplan-Meier e o teste de Breslow. Considerou-se um nível de significância $\alpha=0,05$. A análise estatística dos dados foi efectuada com o programa SPSS ${ }^{\circledR}$ versão 21 (SPSS Inc, Chicago, IL).

\section{RESULTADOS}

Ao longo destes 16 anos (1997 - 2012) foram avaliados nesta Consulta 1042 doentes com infecção por VIH, sendo o período médio de seguimento de 6,5 anos (mínimo 0,003 anos - máximo 16 anos). Destes, foram identificados $33(3,2 \%)$ com o diagnóstico confirmado de Neoplasia Não Definidora de SIDA, cujas características demográficas e clinico-laboratoriais, no que se refere à caracterização da infecção por VIH, se encontra sumarizada na Tabela 1.

A mediana das idades na altura do diagnóstico da infecção pelo VIH foi de 51 anos (23 -75 anos), sendo a maioria do sexo masculino $(78,8 \%)$. A raça caucasiana bem como o $\mathrm{VIH}-1$ foram claramente predominantes (97\% e $93,9 \%$, respectivamente), identificando-se apenas um doente de raça negra (3\%) e 2 casos de infecção por $\mathrm{VIH}-2(6,1 \%)$. Quanto à categoria de transmissão, a via sexual foi a mais frequente, sendo maioritariamente heterossexual $(72,7 \%)$. A representatividade da transmissão associada ao consumo de drogas endovenosas foi de $15,2 \%$.

No que diz respeito ao grau de imunossupressão, e atendendo ao valor dos primeiros linfócitos T CD4 conhecidos, a média foi de $368 \pm 308$ células $/ \mathrm{mm}^{3}$ e a mediana 289 $(19-1500)$ células $/ \mathrm{mm}^{3}$.

No total foram identificados 34 casos de NNDS, dois deles ocorrendo no mesmo doente (neoplasia da bexiga 
Tabela 1 - Características demográficas e clinicopatológicas dos doentes com infecção por VIH e NNDS

\begin{tabular}{|c|c|c|}
\hline Caracteristicas & $n$ & $\%$ \\
\hline $\mathrm{N}^{\circ}$ doentes & 33 & \\
\hline
\end{tabular}

Idade no diagnóstico de VIH (anos)

Mediana

Intervalo

$23-75$

Sexo

Feminino

7

$21,2 \%$

Masculino

26

$78,8 \%$

Raça

Caucasiana

32

$97,0 \%$

Negra

1

$3,0 \%$

Tipo de infecção

VIH 1

31

$93,9 \%$

VIH 2

2

$6,1 \%$

Categoria de transmissão

Heterossexual

24

$72,7 \%$

Toxicodependência

5

$15,2 \%$

Homossexual

3

$9,1 \%$

Desconhecida

1

$3,0 \%$

Exposição a TARV

Sim

24

$72,7 \%$

Sim, mas tempo desconhecido

2

$6,1 \%$

Não

Média de anos até diagnóstico de NNDS $(n=24)$

$6 \pm 3$

Primeiros CD4 (células $/ \mathrm{mm}^{3}$ )

Mediana

Intervalo

$9-1500$

NNDS, neoplasia não-definidora de SIDA; TARV, terapêutica anti-retroviral

e carcinoma basocelular do nariz). De referir ainda o caso de um doente cujo diagnóstico primário de uma neoplasia definidora de SIDA (sarcoma de Kaposi cutâneo) levou ao estadiamento e diagnóstico precoce de uma NNDS (neoplasia do pulmão) - doente $\mathrm{n}^{\circ} 7$, Tabela 2 .

O tempo médio de evolução da infecção por VIH até ao diagnóstico de NNDS foi de 6,8 \pm 4 anos, superior ao tempo decorrido entre a data da primeira consulta e o diagnóstico de NNDS (média 4,6 \pm 4 anos). Houve dois casos cujo diagnóstico de NNDS foi efectuado previamente, noutra instituição de saúde, ambos 3 anos após o diagnóstico de infecção por VIH (doentes n 19 e 22, Tabela 2).

$\mathrm{Na}$ altura do diagnóstico da NNDS os doentes tinham uma idade mediana de 55 anos (31 - 73 anos). O doente mais novo no qual foi diagnosticado um linfoma de Hodgkin escleronodular em estádio IV caracterizava-se por ter um seguimento em consulta irregular, com muito má adesão à terapêutica. Na altura, e apesar de apenas ter infecção VIH-1 conhecida com dois anos de evolução, encontrava-se já muito imunodeprimido (linfócitos TCD4 de 55 células/ $\mathrm{mm}^{3}$ ) e com carga viral do VIH-1 detectável (113 196 cópias $/ \mathrm{ml}, 5,5 \mathrm{log})$ - (doente $\mathrm{n}^{\circ} 26$, Tabela 2). 


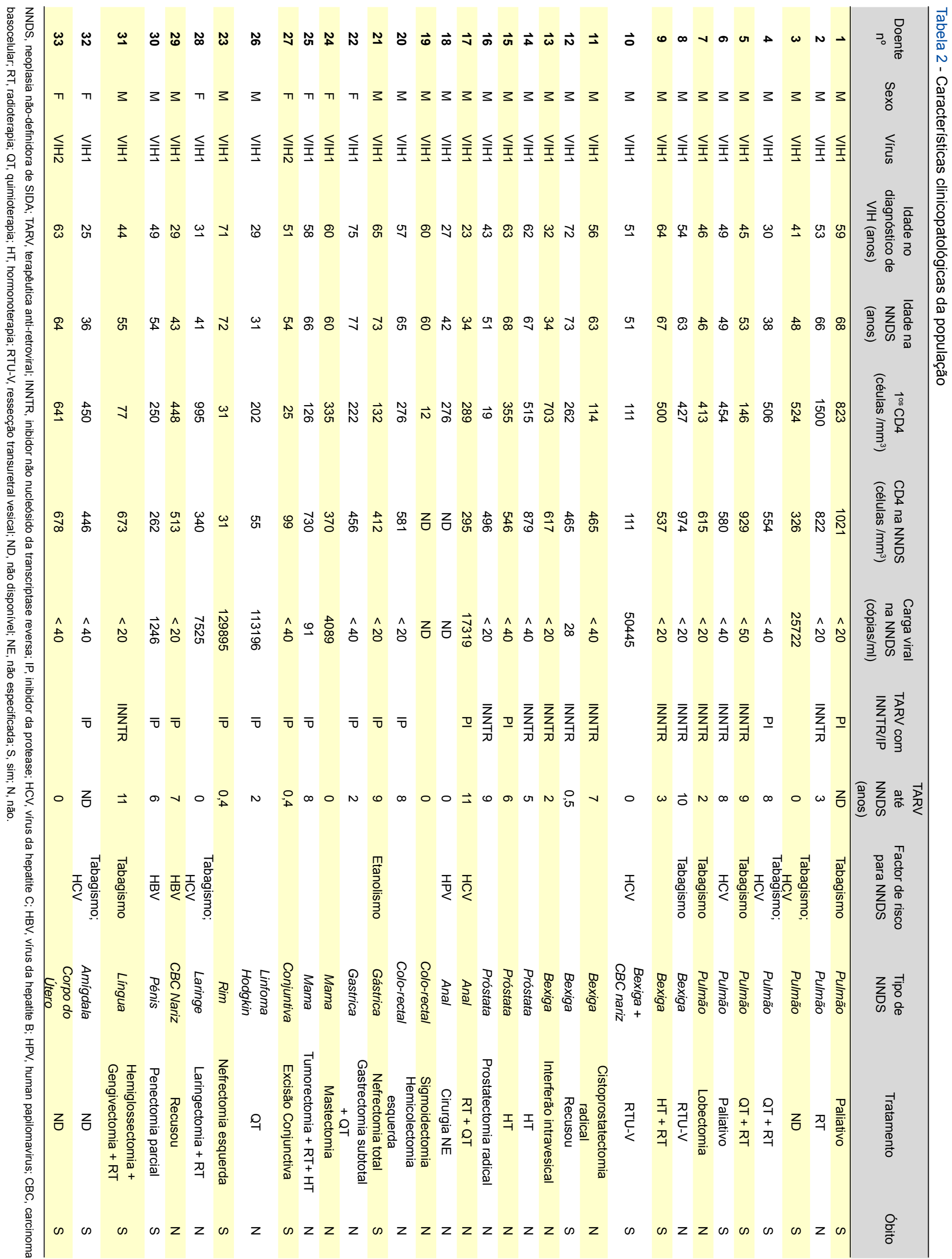


Tabela 3 - Tipos de neoplasias-não-definidoras de SIDA identificadas

\begin{tabular}{|c|c|c|}
\hline Tipo de neoplasia & $n$ & $\%$ \\
\hline Pulmão & 7 & $20,6 \%$ \\
\hline Adenocarcinoma de não pequenas-células & 2 & \\
\hline Carcinoma pavimento-celular & 2 & \\
\hline Carcinoma de pequenas células & 1 & \\
\hline Não especificado & 2 & \\
\hline Bexiga & 6 & $17,6 \%$ \\
\hline Carcinoma invasivo de células de transição & 4 & \\
\hline Carcinoma papilar urotelial de baixo grau & 1 & \\
\hline Carcinoma in situ & 1 & \\
\hline Próstata & 3 & $8,8 \%$ \\
\hline \multicolumn{3}{|l|}{ Adenocarcinoma } \\
\hline Anal & 2 & $5,9 \%$ \\
\hline \multicolumn{3}{|l|}{ Carcinoma pavimento-celular } \\
\hline Mama & 2 & $5,9 \%$ \\
\hline Carcinoma ductal invasivo & 1 & \\
\hline Carcinoma lobular invasivo & 1 & \\
\hline Colo-rectal & 2 & $5,9 \%$ \\
\hline \multicolumn{3}{|l|}{ Adenocarcinoma bem diferenciado } \\
\hline Gástrico & 2 & $5,9 \%$ \\
\hline \multicolumn{3}{|l|}{ Adenocarcinoma } \\
\hline Pele & 2 & $5,9 \%$ \\
\hline \multicolumn{3}{|l|}{ Carcinoma basocelular } \\
\hline Conjuntiva & 1 & $2,9 \%$ \\
\hline \multicolumn{3}{|l|}{ Carcinoma pavimento-celular invasivo } \\
\hline Linfoma de Hodgkin & 1 & $2,9 \%$ \\
\hline \multicolumn{3}{|l|}{ Escleronodular estadio IV } \\
\hline $\operatorname{Rim}$ & 1 & $2,9 \%$ \\
\hline \multicolumn{3}{|l|}{ Carcinoma papilar de células renais } \\
\hline Laringe & 1 & $2,9 \%$ \\
\hline \multicolumn{3}{|l|}{ Carcinoma pavimento-celular } \\
\hline Pénis & 1 & $2,9 \%$ \\
\hline \multicolumn{3}{|l|}{ Carcinoma pavimento-celular } \\
\hline Língua & 1 & $2,9 \%$ \\
\hline \multicolumn{3}{|l|}{ Carcinoma pavimento-celular invasivo } \\
\hline Amígdala & 1 & $2,9 \%$ \\
\hline \multicolumn{3}{|c|}{ Carcinoma pavimento-celular moderadamente diferenciado } \\
\hline Corpo do últero & 1 & $2,9 \%$ \\
\hline \multicolumn{3}{|l|}{ Não especificado } \\
\hline TOTAL NNDS & 34 & $100,0 \%$ \\
\hline $\mathrm{N}^{\circ}$ total de doentes & 33 & \\
\hline
\end{tabular}

NNDS, neoplasia não-definidora de SIDA 
Do ponto de vista imunológico os doentes apresentavam uma média de linfócitos T CD4 de $512 \pm 254$ células/ $\mathrm{mm}^{3}$, estando a maioria $(64 \%)$ controlada e com virémia indetectável.

Nesta altura 26 doentes $(78,8 \%)$ estavam sob TARV, 24 dos quais, em média, desde há $5,7 \pm 3$ anos. No total de 26 doentes, $54 \%$ estavam com um esquema terapêutico que incluia inibidores da protease (IP) e $46 \%$ estavam sob INNTRs (Tabelas 1 e 2).

No que diz respeito ao tipo de NNDS identificadas, a neoplasia do pulmão foi a mais frequente ( 7 casos, $20,6 \%$ ) - a maioria em doentes fumadores $(71,4 \%)$ - seguida da neoplasia da bexiga $(17,6 \%)$, próstata $(8,8 \%)$, e canal anal $(5,9 \%)$. As restantes neoplasias e tipos histológicos encontram-se especificados na Tabela 3.

Relativamente ao estadio da neoplasia na altura do seu diagnóstico, salienta-se que quatro doentes (12\%) apresentavam já doença avançada, particularmente os casos de neoplasia do pulmão e gástrica. Nestes a doença apresentou-se com evidência de metastização à distância, sobretudo hepática, mas também cerebral, óssea e renal (Tabela 4).

Nove doentes estavam co-infectados com HCV (7) ou HBV (2), e houve um caso de infecção por HPV, o qual desenvolveu uma neoplasia do canal anal. Como possíveis factores de risco adicionais foram identificados: hábitos tabágicos $(27 \%)$, hábitos etanólicos $(3 \%)$, consumo de drogas endovenosas $(15,2 \%)$.

O tratamento dos diferentes tipos de neoplasias incluiu: cirurgia (37\%), quimioterapia (QT- 14\%), radioterapia (RT$19 \%$ ) e hormonoterapia (HT - 14\%), isoladamente ou em combinação, conforme representado nas Tabelas 2 e 4. De referir, no entanto, alguns casos pontuais que apenas tiveram indicação para tratamento paliativo (5\%).

No total verificaram-se 15 (45,5\%) óbitos, a maioria dos quais em doentes com neoplasia do pulmão metastizada (20\%) - Fig. 1.

A mediana de idades dos doentes falecidos foi de 56 (36 - 78) anos, sendo a mediana do tempo conhecido de infecção pelo $\mathrm{VIH}$ de oito $(0,5$ - 12) anos. O doente mais jovem faleceu aos 36 anos, no mesmo ano do diagnóstico de um carcinoma espinocelular da amígdala, cujo tratamento se desconhece uma vez que faleceu noutra instituição de saúde. Quanto ao estádio imunovirológico, estes doentes apresentavam uma média de $475 \pm 277$ linfócitos T CD4/ $\mathrm{mm}^{3}, 66,7 \%$ dos quais com carga viral do VIH indetectável. Entre os doentes falecidos contam-se as duas doentes com infecção por VIH-2, no contexto de uma neoplasia da conjuntiva e do corpo do útero, respectivamente.

Como causas de óbito apenas foram apuradas três: a própria neoplasia, um caso de gripe pelo vírus influenzae H1N1, e um doente que faleceu numa Unidade de Cuidados Intensivos no pós-operatório de uma nefrectomia. A restante caracterização dos doentes falecidos encontra-se expressa na Tabela 4

\section{DISCUSSÃO}

As NNDS, patologias de risco acrescido nos doentes com infecção por VIH, constituem actualmente uma importante causa de morbimortalidade nestes doentes. ${ }^{21}$ As mais frequentes são o linfoma de Hodgkin, neoplasia do pulmão, e os carcinomas relacionados com vírus oncogénicos como o carcinoma hepatocelular e neoplasia do canal anal. . $^{1,3,4,9,10}$ Outras cujo risco parece especificamente aumentado nos doentes com infecção VIH são as neoplasias da cabeça e pescoço, colorectal, próstata, pénis e rim. 4,9,10,23

$\mathrm{Na}$ análise realizada, a neoplasia do pulmão foi a mais incidente, seguida da neoplasia da bexiga, próstata e canal anal. As neoplasias da cabeça e pescoço surgiram aqui representadas pelos casos de neoplasia da laringe, língua e amígdala.

No que diz respeito à neoplasia do pulmão, esta constitui actualmente a terceira causa de neoplasia mais frequente nestes doentes, apenas precedida pelas neoplasias definidoras de SIDA como o sarcoma de Kaposi e o linfoma não-Hodgkin. ${ }^{24}$ Tal como na maioria das séries ${ }^{1,12,16,25-27}$ também aqui houve um predomínio no sexo masculino e em doentes fumadores, sendo o adenocarcinoma e o carcinoma pavimento-celular os tipos histológicos mais frequentes, o que aliado a uma tendência para um diagnóstico tardio e já com evidênca de metastização à distância, condicionou um mau prognóstico com alta taxa de mortalidade. Também prevalentes neste estudo foram as neoplasias génito-urinárias (bexiga, próstata e rim). De acordo com revisões anteriores, apesar de a neoplasia da bexiga ser mais prevalente nos doentes imunodeprimidos transplantados, ${ }^{16}$ os dados referentes aos doentes com infecção por VIH são escassos. No entanto, uma idade mais jovem, predomínio no sexo masculino, e queixas de hematúria parecem ser a forma de apresentação mais frequente. Geralmente os doentes caracterizam-se por ter uma imunodepressão apenas moderada, e doença localizada à apresentação, sendo o tipo histológico mais frequente o carcinoma urotelial de células de transição. Como factores de risco associados incluem-se os hábitos tabágicos, parecendo não existir qualquer contributo da utilização de TARV. ${ }^{28}$ No nosso estudo, a forma de apresentação dos diferentes casos de neoplasia da bexiga foi semelhante, à excepção do estadio da doença, mais avançada e com maior número de casos de carcinoma urotelial invasivo. Foram também identificados três casos de neoplasia da próstata e um caso de neoplasia do rim, que à semelhança de estudos anteriores se caracterizaram da mesma forma que na população geral. Neste caso não parece haver qualquer efeito de $\mathrm{VIH}$ nos níveis de PSA, alteração na forma de apresentação da neoplasia, estadiamento ou terapêutica. ${ }^{29}$ De referir no entanto, que alguns estudos apontam para um ligeiro aumento da incidência de neoplasias do rim nesta população, sobretudo o carcinoma de células renais, embora os mecanismos subjacentes não estejam esclarecidos. . $^{1,30,31}$

Também as neoplasias associadas à co-infecção com vírus oncogénicos são mais prevalentes na população infectada por VIH. No caso de HPV, cuja infecção crónica é 


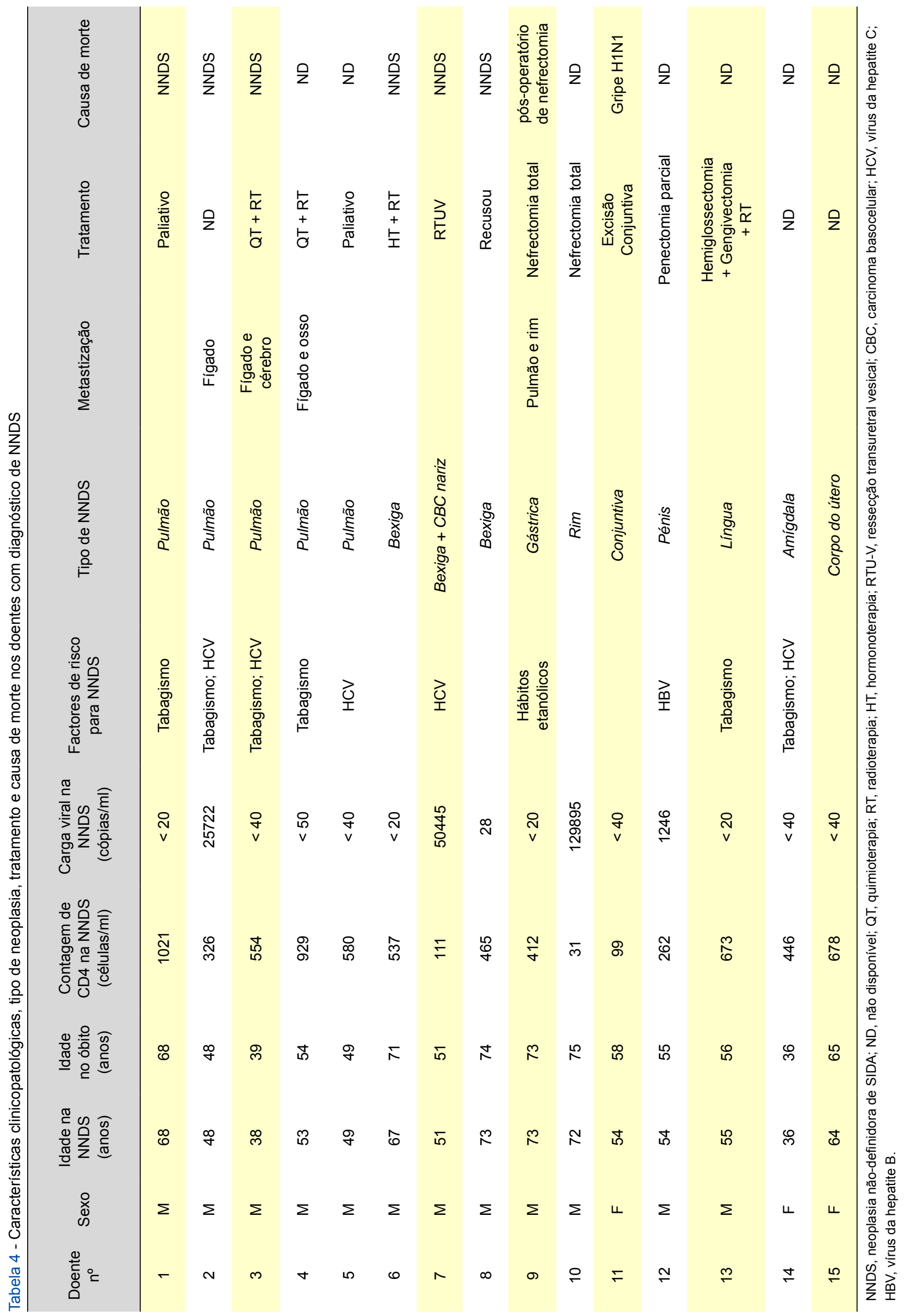




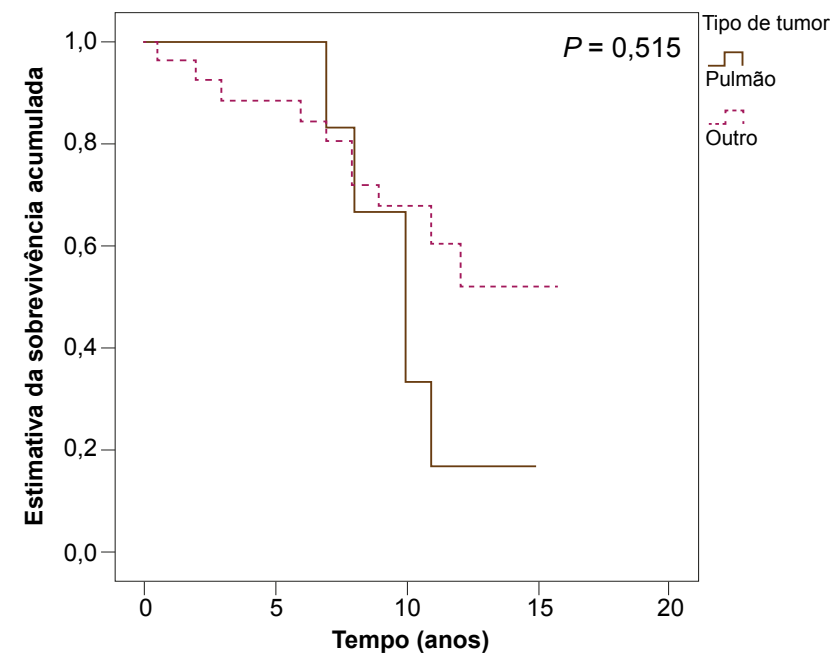

Figura 1 - Estimativa de Kaplan-Meier da função de sobrevivência estratificada por tipo de tumor

facilitada pela infecção por VIH concomitante, este constitui um factor de risco importante para o desenvolvimento de neoplasias do canal anal, pénis e conjuntiva, entre outros. Apesar da neoplasia do canal anal ser pouco frequente na população geral, existe um aumento marcado do risco nos doentes infectados por $\mathrm{VIH}$, particularmente nos homossexuais. ${ }^{7}$ Neste contexto, e apesar de não existirem recomendações especificamente dirigidas a esta população, a realização de uma citologia no momento do diagnóstico de VIH com seguimento semestral até dois testes negativos, além da inspecção e toque rectal anuais, e eventual anuscopia com biópsia se surgirem alterações, poderão estar recomendados. ${ }^{19}$ Neste estudo, detectaram-se dois casos de neoplasia do canal anal, ambos em doentes com comportamento homossexual, jovens, um dos quais co-infectado por HPV. Da mesma forma, a neoplasia do pénis, cujo risco se encontra igualmente aumentado nos doentes co-infectados por VIH e HPV, ${ }^{1}$ constituiu aqui um caso de NNDS.

Apesar da representatividade dos doentes co-infectados por vírus das hepatites B e/ou C (27\%), não se documentou nenhum caso de carcinoma hepatocelular.

De salientar também, que apesar de o linfoma de Hodgkin ser considerado a NNDS mais frequente,$^{12}$ relacionada com a co-infecção por EBV, neste estudo apenas foi detectado um caso. Além disso, apesar do mau prognóstico inerente ao tipo de neoplasia bem como à grave imunossupressão verificada (CD4 55 células/ $\mathrm{mm}^{3}$ ), este doente teve uma boa resposta ao tratamento com QT, mantendo-se em seguimento na nossa consulta 12 anos após o diagnóstico da neoplasia.

Neste estudo foram ainda detectados casos de neoplasia do foro gastrointestinal, nomeadamente neoplasia gástrica e colorectal, ambas mais prevalentes na população infectada por VIH do que na população geral. 2,32,33

De particular interesse são os dois casos de neoplasia, com desfecho fatal, em ambas as doentes infectadas por $\mathrm{VIH}-2$. O primeiro refere-se a um caso de uma neoplasia da conjuntiva, também estreitamente relacionada com a

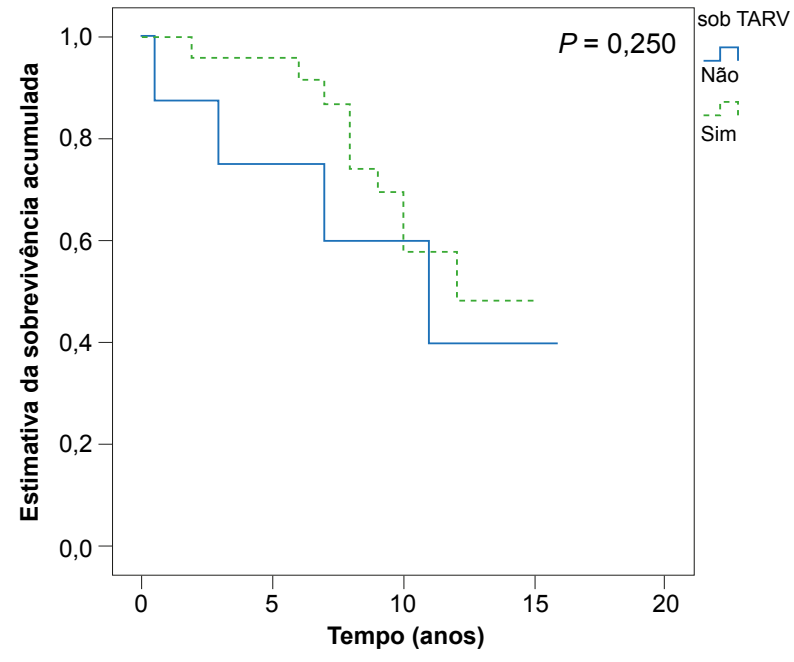

Figura 2 - Estimativa de Kaplan-Meier da função de sobrevivência estratificada por TARV

infecção por HPV e potenciada pela exposição solar e envelhecimento. ${ }^{23}$ Entre as neoplasias relacionadas com HPV esta é considerada uma das mais invasivas nos doentes imunodeprimidos. No entanto é independente da forma de aquisição de $\mathrm{VIH}$, valor de células $\mathrm{CD} 4$, ou tempo de evolução da infecção por VIH subjacente. ${ }^{34}$ Neste caso, tratava-se de uma doente de raça negra, com infecção por VIH-2 conhecida com três anos de evolução, já muito imunodeprimida (CD4 99 células/ $\mathrm{mm}^{3}$ ) e sob TARV com um inibidor da protease (IP), embora com má adesão. Após o diagnóstico da neoplasia com histologia de carcioma pavimento-celular invasivo, foi submetida a excisão da conjuntiva, acabando por falecer quatro anos depois numa Unidade de Cuidados Intensivos no contexto de gripe por vírus influenzae H1N1. O segundo caso diz repeito a uma doente infectada por $\mathrm{VIH}-2$, bem controlada do ponto de vista imunovirológico e sem necessidade de terapêutica, na qual foi feito o diagnóstico de neoplasia do corpo do útero aos 64 anos.

Por fim, salientam-se dois casos de neoplasia da mama. De acordo com a literatura, não é clara a relação causal de $\mathrm{VIH}$ no desenvolvimento dessa patologia. Se alguns estudos apontam para o desenvolvimento de uma doença tendencialmente mais agressiva, preococe e de mau prognóstico, ${ }^{35}$ outros não encontram qualquer diferença na forma de apresentação relativamente à população geral. ${ }^{36}$ Neste estudo, os dois casos de neoplasia surgiram em doentes com 60 e 65 anos de idade, sem evidência de metastização e com boa resposta ao tratamento efectuado.

No que diz respeito aos mecanismos responsáveis pelo aumento da incidência destas neoplasias na população infectada por VIH, os dados são escassos e controversos, sugerindo-se uma etiologia multifactorial.

Atendendo à natureza restrospectiva e observacional deste estudo, bem como à dimensão reduzida da amostra, e ausênca de grupo de controlo (principais limitações deste trabalho) não se podem estabelecer relações de causalidade, nomeadamente no que se refere aos factores de risco oncogénico potencialmente envolvidos. No entanto salien- 


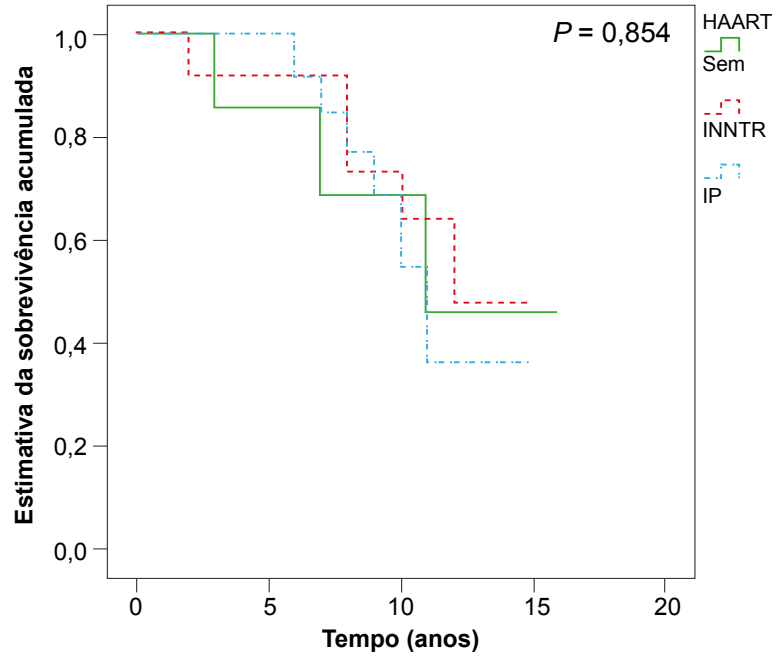

Figura 3 - Estimativa de Kaplan-Meier da função de sobrevivência estratificada por terapêutica

ta-se que a idade jovem na altura do diagnóstico das NNDS (mediana de 55 anos), a incidência de doentes com evidência de metastização na apresentação da NNDS (12\%) bem como a taxa de mortalidade elevada $(45,5 \%)$ detectadas, corroboram os dados da literatura referentes a uma manifestação mais precoce da doença oncológica nos doentes infectados por VIH, de apresentação mais agressiva e com pior prognóstico.

Quanto ao efeito do próprio VIH, grau de imunossupressão e virémia, bem como impacto da utilização de TARV no risco de desenvolvimento de NNDS, a maioria dos doentes encontrava-se imunovirologicamente controlada e sob TARV (54\% IP/ 46\% INNTR). No entanto, não foi feita uma apreciação temporal evolutiva da imunossupressão (sendo apenas incluídas duas determinações por doente) nem dos esquemas terapêuticos prévios, pelo que nada se pode concluir quanto aos efeitos potencialmente carcinogénicos de cada um destes parâmetros (Fig.s 2 e 3). Por outro lado,

\section{REFERÊNCIAS}

1. Frisch M, Biggar RJ, Engels EA, Goedert JJ. Association of cancer with AIDS-related immunosuppression in adults. JAMA. 2001;285:1736-45.

2. Patel P, Hanson D, Sullivan PS, Novak RM, Moorman AC, Tong TC, et al. Incidence of types of cancer among HIV-infected persons compared with the general population in the United States, 1992-2003. Ann Intern Med. 2008;148:728-36.

3. Long JL, Engels EA, Moore RD, Gebo KA. Incidence and outcomes of malignancies in the HAART era in an urban cohort of HIV infected individuals. AIDS. 2008;22:489-96.

4. Crum-Cianflone N, Hullsiek KH, Marconi V, Weintrob A, Ganesan A, Barthel RV, et al. Trends in the incidence of cancers among HIV-infected persons and the impact of antiretroviral therapy: a 20-year cohort study. AIDS. 2009;23:41-50.

5. Palella FJ Jr, Delaney KM, Moorman AC, Loveless MO, Fuhrer J, Satten GA, et al. Declining morbidity and mortality among patients with advanced human immunodeficiency virus infection. HIV Outpatient Study Investigators. N Engl J Med. 1998;338:853-60.

6. Moore RD, Chaisson RE. Natural history of HIV infection in the era of combination antiretroviral therapy. AIDS. 1999;13:1933-42.

7. Clifford GM, Polesel J, Rickenbach M, Dal Maso L, Keiser O, Kofler A, et al. Cancer risk in the Swiss HIV cohort study: associations with immunodeficiency, smoking, and highly Active antiretroviral therapy. J Natl Cancer Inst. 2005;97:425-32.

8. Franceschi S, Lise M, Clifford GM, Rickenbach M, Levi F, Maspoli M, et a presença de determinados comportamentos e estilos de vida como o tabagismo $(27,3 \%)$, os hábitos etanólicos $(3 \%)$ e a co-infecção com vírus oncogénicos (HCV, HBV, HPV) podem ter contribuído para o desenvolvimento de algumas destas neoplasias.

\section{CONCLUSÃO}

Perante o risco de desenvolvimento precoce de diferentes de tipos de neoplasia nos doentes com infecção por $\mathrm{VIH}$, torna-se fundamental o desenvolvimento de estratégias no sentido de diminuir a morbimortalidade que as doenças oncológicas acarretam nesta população. Assim, deve continuar-se a investir na prevenção através de campanhas de informação, medidas gerais como a promoção de cessação tabágica e vacinação, bem como aplicação de protocolos de rastreio ajustados à população com infecção por $\mathrm{VIH}$.

\section{AGRADECIMENTOS}

Ao Serviço de Doenças Infecciosas do Hospital Clinic, Barcelona, em especial a Josep Gatell, Josep Miró e Josep Mallolas.

Ao Grupo da Consulta de Medicina/Imunodeficiência do Serviço de Medicina 1.4 do Hospital de São José - Centro Hospital de Lisboa Central, EPE, particularmente a Isabel Germano, Fátima Lampreia e Umbelina Caixas.

\section{CONFLITOS DE INTERESSE}

A autora declara a inexistência de conflitos de interesse relativamente ao presente artigo. Este trabalho foi apresentado no decurso de realização do "XI Master de SIDA" na Faculdade de Medicina de Barcelona, Espanha.

\section{FONTES DE FINANCIAMENTO}

O trabalho foi realizado sem o suporte de qualquer bolsa ou outro tipo de apoio financeiro.

al. Changing patterns of cancer incidence in the early- and late-HAART periods: the Swiss HIV cohort study. Br J Cancer. 2010;103:416-22.

9. Powles T, Robinson D, Stebbing J, Shamash J, Nelson M, Gazzard B, et al. Highly active antiretroviral therapy and the incidence of non-AIDSdefining cancers in people with HIV infection. J Clin Oncol. 2009;27:88490.

10. Krishnan S, Schouten JT, Jacobson DL, Benson CA, Collier AC, Koletar SL. Incidence of non-AIDS-defining cancer in antiretroviral treatmentnaïve subjects after antiretroviral treatment initiation: an ACTG longitudinal linked randomized trials analysis. Oncology. 2011;80:42-9.

11. Bower M, Collins S, Cottrill C, Cwynarski K, Montoto S, Nelson M, et al. British HIV Association guidelines for HIV-associated malignancies 2008. HIV Med. 2008;9:336-88.

12. Deeken JF, Tjen-A-Looi A, Rudek MA, Okuliar C, Young M, Little RF, et al. The rising challenge of non-AIDS-defining cancers in HIV-infected patients. Clin Infect Dis. 2012;55:1228.

13. Spano JP, Costagliola D, Katlama C, Mounier N, Oksenhendler E, Khayat D. AIDS-related malignancies: state of the art and therapeutic challenges. J Clin Oncol. 2008;26:4834-42.

14. Engels EA, Brock MV, Chen J, Hooker CM, Gillison M, Moore RD. Elevated incidence of lung cancer among HIV-infected individuals. J Clin Oncol. 2006;24:1383-8.

15. Guiguet M, Boué F, Cadranel J, Lang JM, Rosenthal E, Costagliola D. Effect of immunodeficiency, HIV viral load, and antiretroviral therapy on 
the risk of individual malignancies (FHDH-ANRS $\mathrm{CO} 4$ ): a prospective cohort study. Lancet Oncol. 2009;10:1152-9.

16. Grulich $A E$, van Leeuwen MT, Falster MO, Vajdic CM. Incidence of cancers in people with HIVIAIDS compared with immunosuppressed transplant recipients: a meta-analysis. Lancet. 2007:370:59-67.

17. Mitsuyasu RT. Non-AIDS defining malignancies in HIV. Top HIV Med. 2008;16:117-21.

18. Frisch M, Biggar RJ, Goedert JJ. Human papillomavirus-associated cancers in patients with human immunodeficiency virus infection and acquired immunodeficiency syndrome. J Natl Cancer Inst. 2000;92:150010.

19. Tyerman Z, Aboulafia DM. Review of screening guidelines for non-AIDSdefining malignancies: evolving issues in the era of highly active antiretroviral therapy. AIDS Rev. 2012;14:3-16.

20. Gills JJ, Lopiccolo J, Tsurutani J, Shoemaker RH, Best CJ, Abu-Asab MS, et al. Nelfinavir, a lead HIV protease inhibitor, is a broad-spectrum, anticancer agent that induces endoplasmic reticulum stress, autophagy, and apoptosis in vitro and in vivo. Clin Cancer Res. 2007;13:5183-94.

21. Bonnet F, Burty C, Lewden C, Costagliola D, May T, Bouteloup V, et al. Changes in cancer mortality among HIV-infected patients: the Mortalite 2005 Survey. Clin Infect Dis. 2009;48:633-9.

22. European AIDS Clinical Society guidelines 6 [Consultado 2013 Abr 16]. Disponível em http://www.europeanaidsclinicalsociety.org/.

23. Bonnet $F$, Chêne G. Evolving epidemiology of malignancies in HIV. Curr Opin Oncol. 2008:20:534-40.

24. Engels EA, Pfeiffer RM, Goedert JJ, Virgo P, McNeel TS, Scoppa SM, et al. Trends in cancer risk among people with AIDS in the United States 1980-2002. AIDS. 2006;20:1645-54.

25. Kirk GD, Merlo C, O' Driscoll P, Mehta SH, Galai N, Vlahov D, et al. HIV infection is associated with increased risk for lung caner, independent of smoking. AIDS. 2007;45:103-9.

26. Spano JP, Costagliola D, Katlama C, Mounier N, Oksenhendler E, Khayat D. AIDS-related malignancies: state of the art and therapeutic challenges. J Clin Oncol. 2008;26:4834-42.

27. Bonnet F, Lewden C, May T, Heripret L, Jougla E, Bevilacqua S, et al. Malignancy-related causes of death in human immunodeficiency virusinfected patients in the era of highly active antiretroviral therapy. Cancer. $2004 ; 101: 317-24$
28. Gaughan EM, Dezube BJ, Bower M, Aboulafia DM, Bohac G, Cooley TP, et al. HIV-associated bladder cancer: a case series evaluating difficulties in diagnosis and management. BMC Urol. 2009;9:10.

29. Pantanowitz L, Bohac G, Cooley TP, Aboulafia D, Dezube BJ. Human immunodeficiency virus-associated prostate cancer: clinicopathological findings and outcome in a multi-institutional study. BJU Int. 2008;101:1519-23

30. Gaughan EM, Dezube BJ, Aboulafia D, Bower M, Stebbing J, Powles $\mathrm{T}$, et al. Human Immunodeficiency Virus-Associated Renal Cell Carcinoma: a transatlantic case series. Clin Genitourin Cancer. 2008;6:86-90.

31. Baynham SA, Katner HP, Cleveland KB. Increased prevalence of renal cell carcinoma in patients with HIV infection. AIDS Patient Care STDS. 1997;11:161-5.

32. Ford RM, McMahon MM, Wehbi MA. HIVIAIDS and colorectal cancer. A review in the era of antiretrovirals. Gastroenterol Hepatol. 2008;4:274-8.

33. Persson EC, Shiels MS, Dawsey SM, Bhatia K, Anderson LA, Engels EA. Increased risk of stomach and esophageal malignancies in people with AIDS. Gastroenterology. 2012;143:943.

34. Guech-Ongey M, Engels EA, Goedert JJ, Biggar RJ, Mbulaiteye SM. Elevated risk for squamous cell carcinoma of the conjunctiva among adults with AIDS in the United States. Int J Cancer. 2008;122:2590-3.

35. Gewurz BE, Dezube BJ, Pantanowitz L. HIV and the breast. AIDS Read. 2005; $15: 392$.

36. Oluwole SF, Ali AO, Shafaee Z, DePaz HA. Breast cancer in women with HIV/AIDS report of five cases with a review of the literature. J Surg Oncol. 2005;89:23-7.

37. Pantanowitz L, Dezube BJ. Evolving spectrum and incidence of nonAIDS-defining malignancies. Curr Opin HIV AIDS. 2009;4:27-34.

38. Dauby N, De Wit S, Delforge M, Necsoi VC, Clumeck N. Characteristics of non-AIDS-defining malignancies in the HAART era: a clinico-epidemiological study. J Int AIDS Soc. 2011;14:16.

39. Sobhani I, Walker F, Roudot-Thoraval F, Abramowitz L, Johanet H, Hénin $D$, et al. Anal carcinoma: incidence and effect of cumulative infections. AIDS. 2004;18:1561-9.

40. Newton R, Ziegler J, Ateenyi-Agaba C, Bousarghin L, Casabonne D, Beral $\mathrm{V}$, et al. The epidemiology of conjunctival squamous cell carcinoma in Uganda. Br J Cancer. 2002;87:301-8. 


\section{Infecção VIH e Neoplasias Não Definidoras de SIDA: Experiência de um Centro \\ Acta Med Port 2014:27:181-190}

Publicado pela Acta Médica Portuguesa, a Revista Científica da Ordem dos Médicos

Av. Almirante Gago Coutinho, 151

1749-084 Lisboa, Portugal.

Tel: +351 218428215

E-mail: submissao@actamedicaportuguesa.com

www.actamedicaportuguesa.com

ISSN:0870-399X | e-ISSN: 1646-0758

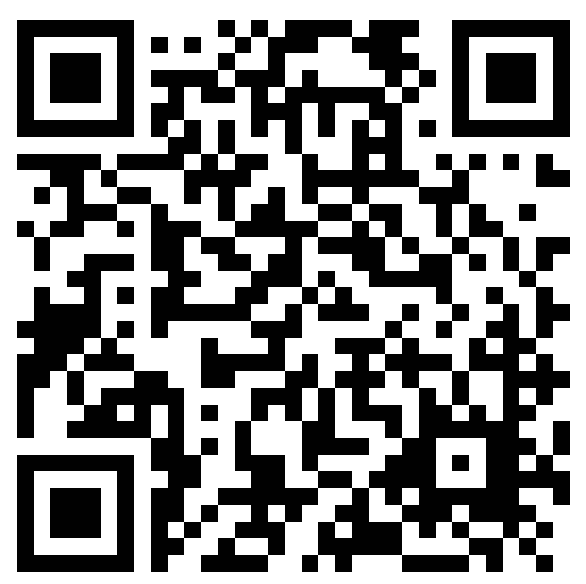

